\title{
RIGID ARTINIAN RINGS
}

\author{
by K. R. McLEAN
}

(Received 29th September 1980)

\section{Introduction}

In [4], Maxson studied the properties of a ring $R$ whose only ring endomorphisms $\phi: R \rightarrow R$ are the trivial ones, namely the identity map, $\mathrm{id}_{R}$, and the map $0_{R}$ given by $\phi(R)=0$. We shall say that any such ring is rigid, slightly extending the definition used in [4] by dropping the restriction that $R^{2} \neq 0$. Maxson's most detailed results concerned the structure of rigid artinian rings, and our main aim is to complete this part of his investigation by establishing the following

Theorem. Let $R(\neq 0)$ be a left-artinian ring. Then $R$ is rigid if and only if

(i) $R \cong Z_{p^{k}}$, the ring of integers modulo a prime power $p^{k}$,

(ii) $R \cong N_{2}$, the null ring on a cyclic group of order 2 , or

(iii) $R$ is a rigid field of characteristic zero.

We shall also show that if $R(\neq 0)$ is any (not necessarily artinian) nilpotent ring, then $R$ is rigid if and only if $R \cong N_{2}$.

From [4] Theorem 3.1, any rigid artinian ring is commutative and is either local or nilpotent. We shall examine these two cases separately.

\section{Rigid artinian local rings}

Let $R$ be a rigid artinian local ring with radical $\boldsymbol{m}$.

Maxson showed that if char $R=0$, then $R$ is a field [4, Theorem 3.2] and that if char $R$ is a prime $p$, then $R \cong Z_{p}$ [4, Corollary 3.2].

The remaining possibility is that char $R=p^{k}$ and char $(R / m)=p$ for some prime $p$ and some integer $k>1$. In this case there exists an unramified complete discrete valuation ring $V$ of characteristic zero whose residue field is isomorphic to $R / m$. This ring $V$ is uniquely determined up to isomorphism by $R / m$ and is called the $v$-ring with residue field $R / \mathbf{m}$. (See [1] Lemma 13, p. 79 and Corollary 2, p. 83.) Moreover, by [1] Theorem 11, p. $79, R$ contains a subring $C$ such that $C \cong V / p^{k} V, C \cap m=p C$ and $R=C+\boldsymbol{m}$. We shall call any such subring $C$ a coefficient ring of $R$.

The following theorem, augmented by Maxson's results, determines the structure of a rigid artinian local ring.

Theorem 1. Let $R$ be a rigid artinian local ring with radical $m$ such that char $R=p^{k}$ and $\operatorname{char}(R / m)=p$ for some prime $p$ and some integer $k>1$. Then $R \cong Z_{p^{k}}$. 
Proof. Since $k>1, \boldsymbol{m} \neq 0$ and we can choose a non-zero element $a \in \boldsymbol{m}^{t-1}$, where $t$ is the index of nilpotence of $\boldsymbol{m}$.

Let $C$ be a coefficient ring of $R$ and $\theta: R \rightarrow R /\left(p R+m^{2}\right)$ be the natural map. We have

$$
p C \subseteq C \cap\left(p R+\boldsymbol{m}^{2}\right) \subseteq C \cap \boldsymbol{m}=p C
$$

and $C / p C \cong R / m$. It follows that $\theta(C)$ is a field.

Suppose that $\theta(R) \neq \theta(C)$. Then there exist elements $x_{1}, \ldots, x_{n} \in R$ such that $\theta(1)$, $\theta\left(x_{1}\right), \ldots, \theta\left(x_{n}\right)$ is a basis for the $\theta(C)$-vector space $\theta(R)$. Now define a map $\phi: R \rightarrow R$ as follows. Given $r \in R$, there are unique elements $\theta\left(c_{i}\right) \in \theta(C)$ such that

$$
\theta(r)=\theta\left(c_{0}\right) \theta(1)+\theta\left(c_{1}\right) \theta\left(x_{1}\right)+\ldots+\theta\left(c_{n}\right) \theta\left(x_{n}\right) .
$$

Lift $\theta\left(c_{1}\right)$ to $c_{i} \in C$. Then $c_{i}$ is unique modulo $p C$, so that $c_{1} a$ is uniquely determined by r. Let

$$
\phi(r)=r+c_{1} a
$$

and check that $\phi$ is a $C$-module homomorphism which acts as the identity on $C$. Since $\boldsymbol{R}=C+\boldsymbol{m}, \phi$ will be a ring endomorphism if $\phi\left(m m^{\prime}\right)=\phi(m) \phi\left(m^{\prime}\right)$ for all $m, m^{\prime} \in \boldsymbol{m}$. But $\theta\left(m^{2}\right)=0$ gives $\phi\left(m m^{\prime}\right)=m m^{\prime}$, whilst $a m=0$ yields $\phi(m) \phi\left(m^{\prime}\right)=m m^{\prime}$. Hence $\phi$ is a ring endomorphism. Now $\phi\left(x_{1}\right)=x_{1}+a$ and $\phi(1)=1$, so that $\phi$ is neither $\operatorname{id}_{R}$ nor $0_{R}$, contradicting the fact that $R$ is rigid.

Thus $\theta(R)=\theta(C)$ and $R=C+p R+m^{2}$. This gives

$$
\boldsymbol{m}=(C \cap \mathbf{m})+\mathrm{p} R+\mathbf{m}^{2}=\mathrm{pC}+\mathrm{pR}+\mathbf{m}^{2}=p R+\boldsymbol{m}^{2} .
$$

Nakayama's lemma yields $\boldsymbol{m}=p R$, whence $R=C+p R$. Using the lemma again, we get $R=C$.

If the residue field $R / \boldsymbol{m}$ where imperfect, it would possess a non-trivial derivation map, $\delta$. Applying [2, Theorem 1], we could lift $\delta$ to a derivation of the $v$-ring $V$ with residue field $R / \boldsymbol{m}$, and hence to a derivation $D$ of $R=C \cong V / p^{k} V$. As in $[3 \S \mathrm{V}]$, the map $\alpha: R \rightarrow R$ given by

$$
\alpha(r)=r+p D(r)+\frac{p^{2}}{2 !} D^{2}(r)+\ldots+\frac{p^{k-1}}{(k-1) !} D^{k-1}(r)
$$

would be a non-trivial automorphism of $R$, contradicting the rigidity of $R$.

Hence $R / m$ is a perfect field and, by [4, Theorem 3.3], $R \cong Z_{p^{k}}$.

\section{Rigid nilpotent rings}

No artinian hypothesis is needed in the next theorem.

Theorem 2. Let $R(\neq 0)$ be a rigid nilpotent ring. Then $R \cong N_{2}$.

Proof. Suppose that $R^{2} \neq 0$. Then $R$ has index of nilpotence $t>2$ and there exists $a \in R^{t-2}$ such that $a R \neq 0$. The map $\phi: R \rightarrow R$ given by $\phi(r)=r+a r$ is a ring endomorphism which is not the identity, but which acts as the identity on the non-zero ideal $R^{2}$. This contradicts the rigidity of $R$.

Hence $R^{2}=0$. By considering the map $r \rightarrow 2 r$ on $R$, it is easy to see that $R \cong N_{2}$. 
Combining Maxson's results with our own, we have the theorem stated in the introduction.

\section{REFERENCES}

1. I. S. Conen, On the structure and ideal theory of complete local rings, Trans. Amer. Math. Soc. 59 (1946), 54-106.

2. N. Heerema, Derivations on p-adic fields, Trans. Amer. Math. Soc. 102 (1962), 346-351.

3. N. Heerema, Higher derivations and automorphisms of complete local rings, Bull. Amer. Math. Soc. 76 (1970), 1212-1225.

4. C. J. Maxson, Rigid rings, Proc. Edinburgh Math. Soc. 21 (1978), 95-101.

SCHOOL OF EDUCATION

UNIVERSITY OF LIVERPOOL

P.O. Box 147

LIVERPOOL L69 3BX 